\title{
Quasars Have Fewer Close Companions than Normal Galaxies
}

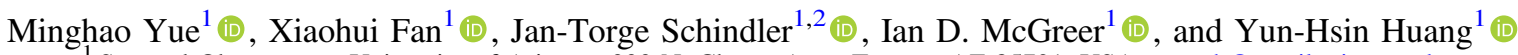 \\ ${ }^{1}$ Steward Observatory, University of Arizona, 933 N. Cherry Ave., Tucson, AZ 85721, USA; yuemh @email.arizona.edu \\ ${ }^{2}$ Max Planck Institute for Astronomy, Königstuhl 17, D-69117 Heidelberg, Germany \\ Received 2019 May 2; revised 2019 August 4; accepted 2019 August 19; published 2019 September 27
}

\begin{abstract}
We investigate the distribution of companion galaxies around quasars using Hubble Space Telescope (HST) Advanced Camera for Surveys Wide Field Camera (ACS/WFC) archival images. Our master sample contains 532 quasars that have been observed by HST ACS/WFC, spanning a wide range of luminosity $\left(-31<M_{i}(z=2)<-23\right)$ and redshift $(0.3<z<3)$. We search for companions around the quasars with a projected distance of $10 \mathrm{kpc}<d<100 \mathrm{kpc}$. Point spread function subtraction is performed to enhance the completeness for close companions. The completeness is estimated to be high $(>90 \%)$ even for the faintest companions of interest. The number of physical companions is estimated by subtracting a background density from the number density of projected companions. We divide all the companions into three groups (faint, intermediate, and bright) according to their fluxes. A control sample of galaxies is constructed to have a similar redshift distribution and stellar mass range as the quasar sample using the data from HST deep fields. We find that quasars and control sample galaxies have similar numbers of faint and bright companions, while quasars show a $3.7 \sigma$ deficit of intermediate companions compared to galaxies. The numbers of companions in all three groups do not show strong evolution with redshift, and the number of intermediate companions around quasars decreases with quasar luminosity. Assuming that merger-triggered quasars have entered the final coalescence stage during which individual companions are no longer detectable at large separations, our result is consistent with a picture in which a significant fraction of quasars is triggered by mergers.
\end{abstract}

Key words: galaxies: active - galaxies: evolution - galaxies: nuclei - quasars: general

Supporting material: machine-readable table

\section{Introduction}

Active galactic nuclei (AGNs) play important roles in not only the growth of supermassive black holes (SMBHs) in the centers of galaxies, but also in the evolution of their host galaxies. SMBHs gain most of their mass through the active galactic nucleus (AGN) phase, and AGN activities can significantly influence the evolution of their host galaxies (for a recent review, see Kormendy \& Ho 2013). AGN can heat up and expel gas from their host galaxies and thus quench star formation (e.g., Croton et al. 2006; Cicone et al. 2014; Spacek et al. 2016). To illustrate the entire SMBH and galaxy evolution through cosmic time, it is crucial to understand the triggering mechanism of AGNs.

Two scenarios have been proposed that can trigger AGN activities: major mergers of galaxies (especially gas-rich ones) and secular evolution. Major mergers can disturb gas in galaxies and generate gas inflows that are needed to feed the central SMBH (e.g., Barnes \& Hernquist 1991; Hopkins et al. 2006). Secular evolution happens when instabilities in galaxies, including those induced by galaxy bars or resulting from the gas inflows from the environment, drive gas to gradually move inward and fuel the SMBH (e.g., Shlosman et al. 1989; Hopkins \& Quataert 2010).

While simulations have shown that both scenarios can lead to rapid SMBH growth in the AGN phase, observational evidence regarding which one is the dominant mechanism remains ambiguous. A crucial test is to measure the merging fraction of their host galaxies (e.g., Grogin et al. 2005; Karouzos et al. 2014). The Hubble Space Telescope (HST) is powerful in identifying galaxy mergers in AGNs because of its small point spread function (PSF). Cisternas et al. (2011) analyzed the morphology of X-ray selected AGNs; they found that their merging fraction is the same as inactive galaxies within measurement errors. Villforth et al. (2017) worked on a sample of more luminous X-ray selected AGNs and reached a conclusion similar to that of Cisternas et al. (2011). However, Fan et al. (2016) reported an enhanced merging fraction of infrared-selected AGNs. Using imaging data from the HyperSupreme Camera Survey (Aihara et al. 2018), which used the Subaru Telescope, Goulding et al. (2018) also showed that the merging fraction of infrared-selected AGNs is larger than that of inactive galaxies. Treister et al. (2012) argued that major mergers are only responsible to the most luminous AGNs. These observational results, together with simulations (e.g., Hopkins et al. 2006; Hopkins \& Quataert 2010), suggest that the two triggering mechanisms may dominate for AGNs of different properties (e.g., luminosity, obscuration, or redshift).

The majority of previous studies used disturbed galaxy morphology to identify recent merging events. Galaxy pairs, or close companions of galaxies, is another frequently used indicator of galaxy mergers (e.g., Man et al. 2012). Ellison et al. (2011) measured the "pair fraction" (i.e., the average number of close companions) of 11,060 galaxies from the Sloan Digital Sky Survey (SDSS), claiming that the merging fraction of emission-line-selected low-luminosity AGNs is larger than that of the galaxy control sample.

Although there have been a number of previous studies to constrain the merging fraction of AGNs, few of them focused on the most luminous population of AGNs, i.e., type-1 quasars. Unlike the low-luminosity AGNs, quasars are usually much brighter than their host galaxies, which makes it very difficult to detect the disturbed features in quasar host galaxies using ground-based imaging. HST imaging can resolve some quasar 
host galaxies, mostly at low redshift $(z \lesssim 1)$. However, the sample sizes of studies based on HST are small, usually containing several tens of AGNs, resulting in large statistical errors. On the other hand, using close companions as indicators of mergers is more accessible than disturbed host galaxy morphology for bright quasars, and can be expanded to larger samples. However, systematic studies on luminous quasar companions using $H S T$ are still lacking.

In this work, we measure the statistics of quasar companions and use the result to constrain the merging fraction of quasars. We use $H S T$ archival imaging for companion detection. Our master sample contains 532 quasars, which is much larger than previous studies based on HST imaging. The paper is organized as follows. Section 2 describes the selection of the quasar sample and archival images. Section 3 describes the detection of close companions, including the PSF-subtraction method, as well as measurements of quasar companion fractions. The selection of the galaxy control sample and the comparison between companion fractions in quasars and normal galaxies are discussed in Section 4. Section 5 discusses the implication of the companion fraction in quasars in the context of the merger-driver model of quasar triggering. Section 6 summarizes the paper. We use AB magnitude through this work, as well as a Lambda cold dark matter cosmology with $\Omega_{\mathrm{M}}=0.3$, $\Omega_{\Lambda}=0.7$ and $H_{0}=70 \mathrm{~km} \mathrm{~s}^{-1}$.

\section{The Quasar Sample}

Our input parent quasar sample is based on the Véron Catalog of Quasars and AGN, 13th edition (Véron-Cetty \& Véron 2010, hereafter the Véron Catalog), the SDSS Data Release (DR7) (Schneider et al. 2010), DR12 (Pâris et al. 2017) and DR14 (Pâris et al. 2018) quasar catalogs. SDSS quasar catalogs provide $i$-band absolute magnitudes that are $K$ corrected to $z=2\left(M_{i}(z=2)\right.$, see Richards et al. 2006) of quasars, and the Véron Catalog provide $B$-band absolute magnitude $\left(M_{B}\right)$. We convert $M_{B}$ of quasars from the Véron Catalog to $M_{i}(z=2)$, according to the relation in Richards et al. (2006). Quasars that have $M_{i}(z=2)<-23$ are selected as our parent sample. We further exclude $z<0.3$ objects to avoid AGNs with extended emission, which are confusing in PSF subtraction, and $z>3$ objects because our control sample becomes incomplete at $z>3$ (see Section 4.1 for details).

We use archival broadband images of the Advanced Camera for Surveys Wide Field Camera (ACS/WFC) for quasar companion detection. The reason to use ACS/WFC images is the small PSF size and large field of view (FoV). A small PSF is crucial for detecting companions that are very close to bright quasars, and a large FoV ensures a valid estimation for the number density of foreground and background objects. The ACS/WFC images that contain the selected quasars are retrieved from the Hubble Legacy Archive (HLA). ${ }^{3}$ These images are generated by the HLA using DrizzlePac tools (Gonzaga et al. 2012), which combine raw exposures with the same filter, same camera, and within the same visit. For each quasar, we choose the deepest image in each band to form our master image sample. In total, 595 quasars are found to appear in 806 images at this step. We run SExtractor (Bertin \& Arnouts 1996) on each image to generate a source catalog.

Though image coaddition can enhance the depth of images, we do not perform image coaddition because it will introduce

\footnotetext{
https://hla.stsci.edu/
}

Table 1

The Number of Images Excluded by the Selection Criteria

\begin{tabular}{lcc}
\hline \hline Criteria & Number of Images & Number of Quasars \\
\hline Total & 806 & 595 \\
\hline Lensed & 6 & 5 \\
NCOMBINE $=1$ & 32 & 30 \\
Close the edge & 25 & 23 \\
Crowded & 63 & 45 \\
\hline All bad images & 126 & 98 \\
\hline Good images & 687 & 532 \\
\hline
\end{tabular}

Note. There are overlaps between different subsets of images/quasars. For example, one quasar may appear in both bad images and good images. As a result, the total number of quasars does not equal to the number of quasars in "ood" images plus those in "bad" images.

difficulty to the background object number density estimation. In most cases, the overlapping area of different images containing the same quasar is a small part of the original images. The number density of background/foreground objects is estimated based on the number of objects in the whole image (see Section 3 for details). Most coadded images do not have enough area to perform a reliable background object density estimation.

We measure the statistics of quasar companions by counting all the projected companions and subtracting a background object density. We thus exclude all quasars that are strongly lensed, because the lensed images can be confusing when counting companions. We further exclude all images that satisfy any of the following:

1. Images with NCOMBINE $=1$, where NCOMBINE is the "NCOMBINE" parameter in the header of the HST image, representing the number of images used for cosmic ray rejection when combining raw exposures. Images with NCOMBINE $=1$ are severely polluted by cosmic rays and are not suitable for companion counting.

2. Images where the target quasar is located close (less than $\left.2^{\prime \prime}\right)$ to the edge of the CCD.

3. Images that are very crowded. The statistical errors on background object density are high. Images which have more than 5000 objects that were brighter than 25 mag in the observed band are excluded in the further analysis.

We summarize the number of images excluded by each criterion in Table 1. All the images that remain after the selection are referred to as "good" images in rest of the paper. The final master sample contains 532 quasars in 687 good images. Among the 532 quasars, 402 of them were observed in programs that were not related to AGN studies. The fact that most quasars were observed by chance ensures a small selection effect (see Section 5.1 for further discussion). In the master sample, one quasar might show up in multiple bands, but there will only be one image of the quasar given a certain filter. Figure 1 shows the redshift and luminosity distribution of the quasars, and Figure 2 shows the number of quasars observed in each band. Images in the F814W band dominate the sample.

When studying quasar companions of certain magnitude, we will only analyze images that are deep enough to detect the faintest companions of interest at a $5 \sigma$ level. Previous studies 


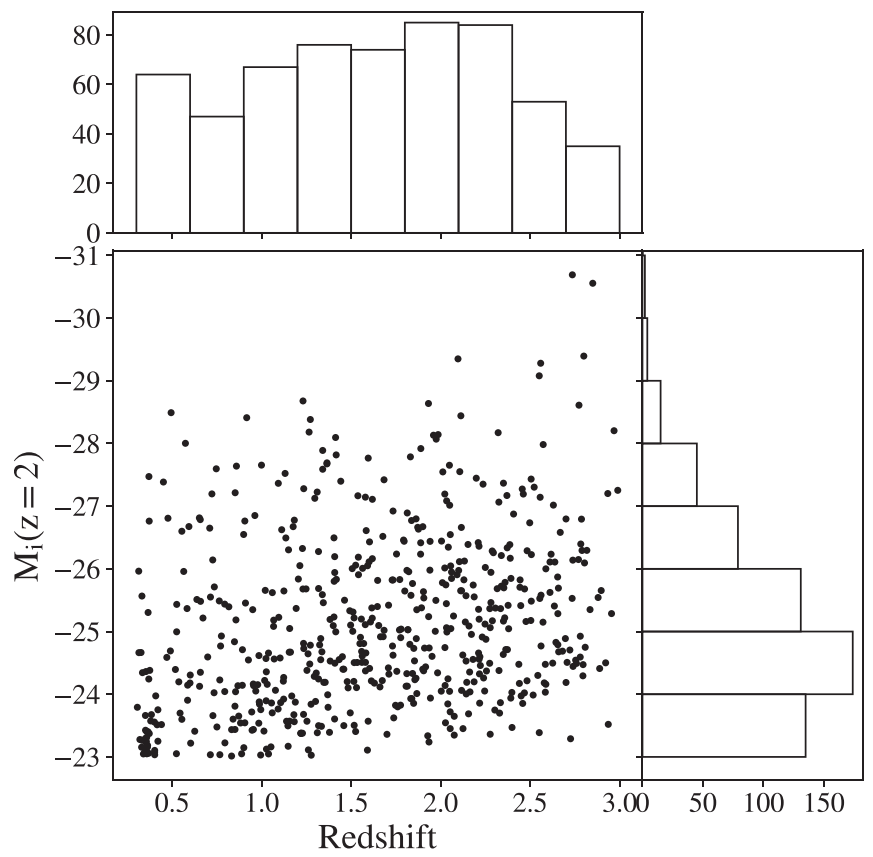

Figure 1. The redshift and luminosity distributions of the quasars in our master sample. This sample contains 532 quasars that show up in 687 HST ACS/WFC archival images.

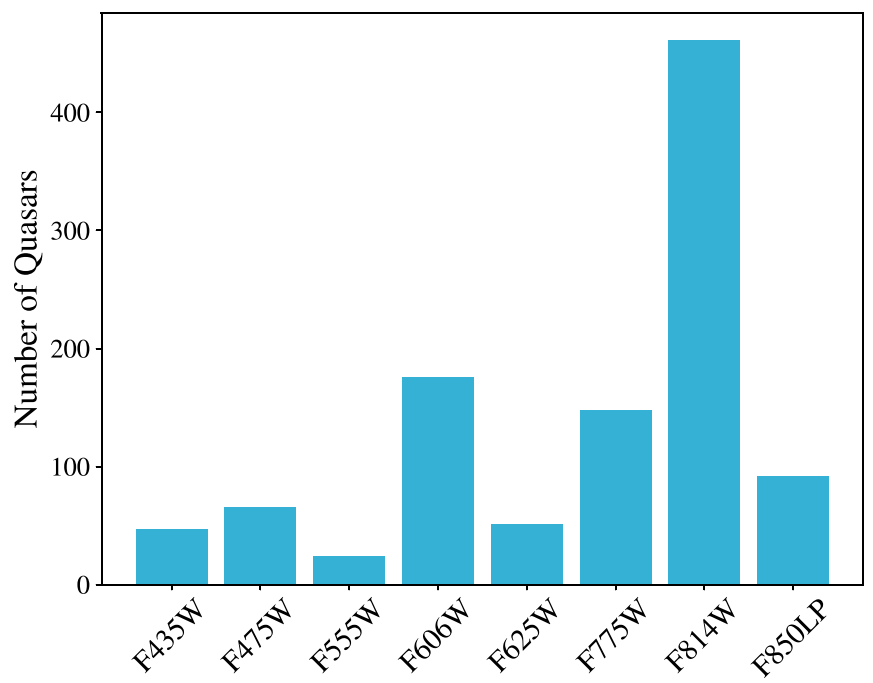

Figure 2. Number of quasars observed in each band. Note that one quasar can be observed by multiple bands.

(e.g., Matsuoka et al. 2014) showed that quasar host galaxies have a typical stellar mass range $M_{*} \gtrsim 10^{10} M_{\odot}$ and a typical luminosity $M_{g} \lesssim-21$ in the SDSS $g$-band. We are mainly interested in companions that have stellar masses close to the quasar host galaxy, which correspond to major mergers. We use two sets of magnitude limits:

1. A "simple" cut on absolute magnitude in each band. Companions with $M_{\mathrm{abs}}<-19$ will be analyzed, regardless of in which band the quasar was observed. This sample is constructed as a "pure" observational result that can be directly compared with simulations without any further assumption. We convert the absolute magnitude $M_{\mathrm{abs}}=-19$ to an apparent magnitude $m\left(M_{\mathrm{abs}}=-19\right)$, assuming that the companions have the same redshift as the quasar, and require that the image is deep enough to
Table 2

Summary of Samples Used in this Study

\begin{tabular}{llc}
\hline \hline Sample & Description $^{\text {a }}$ & Number of Quasars \\
\hline Master & The master sample & 532 \\
\hline A & $\begin{array}{l}\text { The image is deep enough to } \\
\text { detect an object of absolute } \\
\text { magnitude } M_{\text {abs }}=-19 \text { at } \\
\text { the quasar's redshift. }\end{array}$ & \\
\hline B & $\begin{array}{l}\text { The image is deep enough to } \\
\text { detect an object of apparent } \\
\text { magnitude } m=m\left(10^{9} M_{\odot}\right) .\end{array}$ & 354 \\
&
\end{tabular}

Notes.

${ }^{\text {a }}$ All magnitude limits are for point sources at $5 \sigma$ level.

b The expression $m\left(M_{*}\right)$ refers to the apparent magnitude corresponding to stellar mass $M_{*}$. See Section 4.1 for the detailed definition.

detect an object as faint as $m\left(M_{\text {abs }}=-19\right)$. We do not perform a $K$-correction when converting $M_{\text {abs }}$ to $m\left(M_{\text {abs }}\right)$, because most quasars were observed in only one band. If a quasar is observed in multiple bands, the deepest image relative to $M_{\mathrm{abs}}=-19$ is used. Specifically, the depth of an image relative to $M_{\mathrm{abs}}=-19$ (regardless of the filter) is quantified by $m_{\mathrm{lim}}-m\left(M_{\mathrm{abs}}\right)$, where $m_{\mathrm{lim}}$ stands for the $5 \sigma$ depth of the image. The quasar sample corresponding to this magnitude cut is referred to as "sample A." The analysis of this sample will be described in Section 3.

2. A cut based on stellar masses of companions. We convert stellar masses $\left(M_{*}\right)$ to observed magnitudes (denoted by $m\left(M_{*}\right)$ ) using abundance matching (see Section 4.1 for details). This sample is constructed to enable physical interpretations of our result. Companions brighter than $m\left(10^{9} M_{\odot}\right)$ will be analyzed. Similar to Sample A, if a quasar is observed in multiple bands, the deepest image relative to $m\left(10^{9} M_{\odot}\right)$ will be used. The quasar sample corresponding to this magnitude cut is referred to as "Sample B." The analysis of this sample will be described in Section 4.

The properties of the two quasar samples, together with the master sample, are summarized in Table 2. Unlike in the master sample where a quasar may show up in multiple images, in Samples A and B, one quasar only shows up in one image.

\section{Detecting Close Companions of Quasars by PSF Subtraction}

In this section, we will describe our PSF-subtraction and companion detection method. We will also discuss the statistics of quasar companions with absolute magnitude $M_{\mathrm{abs}}<-19$, regardless of the band in which the quasar image was taken. Accordingly, we use Sample A throughout this section.

\subsection{Method}

We use close companions of quasars to trace the merging history of quasar host galaxies. If the AGN activity appears in a certain stage of a galaxy merger, the number of companions around quasars will be different from that of inactive galaxies. Specifically, if two merging galaxies are still distinguishable when the quasar appears, we will see a pair of galaxies in the field; if the quasar emerges when the two progenitor galaxies 
have already merged into one galaxy, we will see one single quasar host galaxy rather than a pair. We will discuss this point in Section 5.3 in more detail.

We first perform PSF subtraction to suppress the influence of quasar light on detecting their companions. The PSF model is generated by TinyTim (Krist et al. 2011). TinyTim takes the observation time, the position on the CCD chips and the spectrum shape of the source as input parameters. We use a power-law spectrum with a power-law index of $-2\left(F_{\lambda} \propto \lambda^{-2}\right)$ as the input spectrum to mimic the typical spectral energy distribution (SED) of a quasar. We use a modeled PSF rather than an empirical PSF because most quasars in our sample come from programs in which the quasars are not the primary science targets, and no separate PSF star observations are available. In addition, the wings of bright quasars can make it difficult to detect projected companions that are several arcseconds away from these quasars, thus a large PSF image is preferred. A modeled PSF can be as large as $20^{\prime \prime}$, which is difficult for an empirical PSF. The size of the PSF models used in this study is $20^{\prime \prime} \times 20^{\prime \prime}$.

We use GALFIT (Peng et al. 2002) to perform PSF subtraction. Quasar images are fitted by a PSF component plus a Sérsic profile (Sérsic 1963) for the host galaxy. Examples of PSF subtraction can be found in the Appendix. We run SExtractor on the PSF-subtracted images and select all objects with a projected distance to the quasar of $10 \mathrm{kpc}<d<100 \mathrm{kpc}$ as projected quasar companions. We divide the projected distance range $10 \mathrm{kpc}<d<100 \mathrm{kpc}$ into 9 bins, with the $i$ th bin at $10 \times i \mathrm{kpc}<d<10 \times(i+1) \mathrm{kpc}$. The region where $d<10 \mathrm{kpc}$ is severely influenced by the PSFsubtraction residuals for many quasars and is not analyzed.

Companions selected in this way will be inevitably contaminated by foreground and background objects. In the following text, we use "projected companions" to represent all the companions detected (both physical and unphysical). To estimate the numbers of physical companions, a "background density" representing the background/foreground object surface density is calculated for each quasar. We use the entire image to estimate the background object density. Specifically, the surface number density of physical companions of the $i$ th bin is estimated by

$$
\sigma_{\mathrm{phys}}\left(d_{i}, d_{i+1}\right)=\frac{N\left(d_{i}, d_{i+1}\right)}{A\left(d_{i}, d_{i+1}\right) C\left(d_{i}, d_{i+1}\right)}-\frac{N_{\mathrm{bkg}}}{A_{\mathrm{bkg}}},
$$

where $N\left(d_{i}, d_{i+1}\right)$ stands for the number of projected companions with $10 \times i \mathrm{kpc}<d<10 \times(i+1) \mathrm{kpc}, A\left(d_{i}, d_{i+1}\right)$ is the corresponding area, and $C\left(d_{i}, d_{i+1}\right)$ is the completeness of companion detection (see Section 3.2 for details). $N_{\mathrm{bkg}}$ and $A_{\mathrm{bkg}}$ describe the numbers of objects and the area of the whole image. The number of physical companions with $10 \times i \mathrm{kpc}<d<$ $10 \times j \mathrm{kpc}(1 \leqslant i<j \leqslant 9)$ is calculated by summing up the number of physical companions in the corresponding bins:

$$
N_{\text {phys }}\left(d_{i}, d_{j}\right)=\sum_{i \leqslant k<j} \sigma_{\text {phys }}\left(d_{k}, d_{k+1}\right) A\left(d_{k}, d_{k+1}\right),
$$

and the error of $N_{\text {phys }}\left(d_{i}, d_{j}\right)$ is estimated assuming a Poisson distribution for $N\left(d_{i}, d_{i+1}\right)$ and $N_{\mathrm{bkg}}$ in Equation (1).

Unless specified, in the rest of the paper, "number of companions" refers for the estimated number of physical companions and "distance" means projected distance.

\subsection{Companion Detection Completeness}

Some companions may be missed or misidentified as a result of imperfect PSF subtraction because it can be difficult to distinguish companions from the PSF-subtraction residuals. The probability of a companion to be detected is mainly influenced by the flux contrast between the companion and the PSF-subtraction residual. We find four factors that have a major influence on the completeness: (1) the flux of the quasar, (2) the accuracy of the PSF models, (3) the angular distance from the quasar to the companion, and (4) the flux of the companion. Factors (1) and (2) vary from quasar to quasar, while factors (3) and (4) are determined by the companion itself. Accordingly, we add simulated companions with different flux and distance for each quasar image to estimate the fraction of missed companions. The simulated companions are generated to be point sources. For each quasar image, we simulate three sets of companions, with absolute magnitude $M_{\mathrm{abs}}=-19,-20,-21$ in the corresponding band, assuming the companions to have the same redshift as the quasar. The completeness is estimated as a function of projected distance to the quasar. We generate 10 simulated companions at randomized positions in each distance bin. The simulated images are analyzed by the companion detection process described above, according to which we calculate the completeness of the companion detection for each quasar. A simulated companion is regarded as "detected" if an object is detected within 0!" 2 from the position of the simulated companion.

In the simulation, we do not consider false positives resulting from the PSF-subtraction residual because the probability for a PSF-subtraction residual to appear right at the position of a simulated companion is negligible. The simulation ensures that most companions of interest can be detected. To exclude false positives in the real images, we visually inspect all the images and remove suspicious detections that are likely PSF-subtraction residuals.

Figure 3 shows the average completeness as a function of companion flux and the distance to the quasar from our simulation. The completeness is larger than $90 \%$ even for the faintest companion in the smallest distance bin $(10 \mathrm{kpc}<$ $d<20 \mathrm{kpc}$ ). For companions that are more than $50 \mathrm{kpc}$ away from the quasar (which corresponds to $\sim 6^{\prime \prime}$ at $z=1.6$ ), the completeness does not change with the distance, which indicates that the influence of the quasar light becomes negligible at larger distances.

We test the potential influence of using point sources as simulated companions. We run another simulation where the shape of the companions are exponential disks with an effective radius of $r_{e}=2 \mathrm{kpc}$, assuming that the companion has a same redshift as the quasar. The difference between the completeness given by the two simulations is less than $1 \%$.

\subsection{Quasar Companion Fraction ${ }^{4}$}

Here we examine the statistics of quasar companions with $-20<M_{\mathrm{abs}}<-19,-21<M_{\mathrm{abs}}<-20$, and $M_{\mathrm{abs}}<-21$, regardless of the band in which the quasar was observed. We do not perform $K$-corrections on the magnitudes. Figure 4 shows the average number of companions around quasars in Sample A. The number is negative in some distance ranges due to the subtraction of background object number density.

\footnotetext{
4 The information of all the quasars and quasar companions is available at (https://github.com/yuemh/qso_companion).
} 


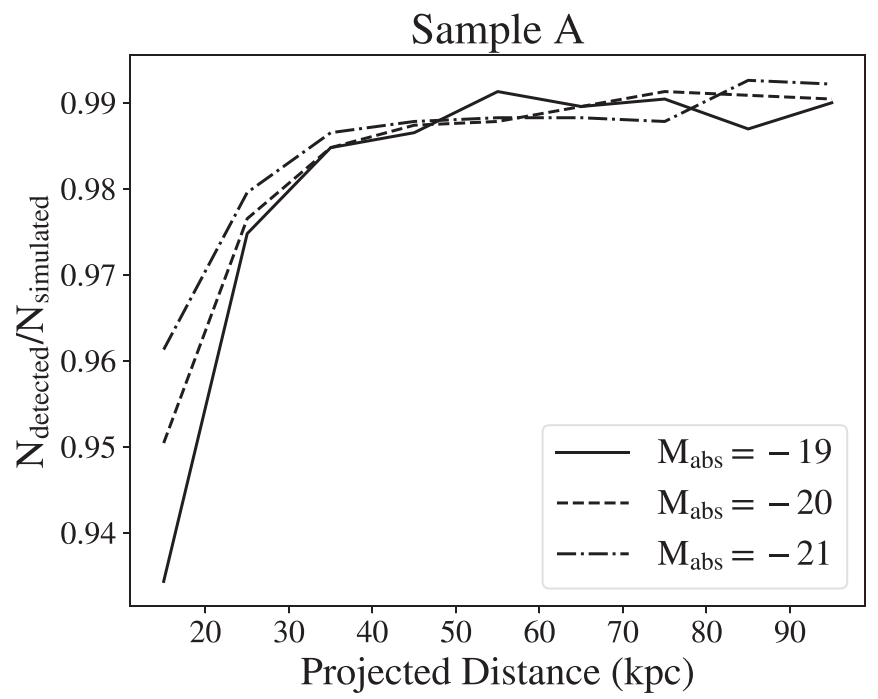

Figure 3. The completeness of companion detection estimated by simulated companions. The simulated objects are generated as point sources. We estimated that the fraction of missed companions is less than $10 \%$ for all the companions of interest.

We define companions with a projected distance of $10 \mathrm{kpc}<$ $d<30 \mathrm{kpc}$ to quasars as "close companions" and calculate the average number of close companions $\left(\bar{N}_{\text {comp }}\right)$ around quasars (galaxies). The distance range is chosen because companions at $10 \mathrm{kpc}<d<30 \mathrm{kpc}$ are likely involved in a merging event, while companions at larger distances are not necessarily associated with mergers. A similar distance range has been adopted in previous studies on the galaxy merging rates (e.g., Lambas et al. 2003; Ellison et al. 2008; de Ravel et al. 2009; Man et al. 2012). The average numbers of close physical companions are $0.26 \pm 0.05,0.06 \pm 0.03$, and $0.05 \pm 0.03$ for $-20<M_{\text {abs }}<-19,-21<M_{\text {abs }}<-20$, and $M_{\text {abs }}<$ -21 companions, and $0.38 \pm 0.07$ for all physical companions which have $M_{\text {abs }}<-19$.

One issue of this analysis is that the "absolute magnitude" we use here is difficult to be translated to physical properties of companions, given that we do not perform $K$-corrections on these magnitudes. $K$-corrections require knowledge of the companion SED, while we usually have only one band measurement. This issue will be addressed in Section 4, by introducing a magnitude cut which is associated with galaxy stellar masses.

\subsubsection{Ongoing Merging Systems}

In addition to statistical studies of large samples, detailed modeling and observations of individual cases are also crucial to the understanding of the quasar triggering mechanism. Here we report some ongoing mergers with quasar activity. Followup observations on these objects, such as host galaxy morphology, gas kinetics, and AGN obscuration can be compared directly with simulations. We visually inspect images of quasars in the master sample, and select objects that show disrupted features like tidal tails and asymmetric host galaxies. We find 22 quasars with features of recent mergers. The images of these objects can be found in the Appendix. Note that these objects are included in both Samples A and B.

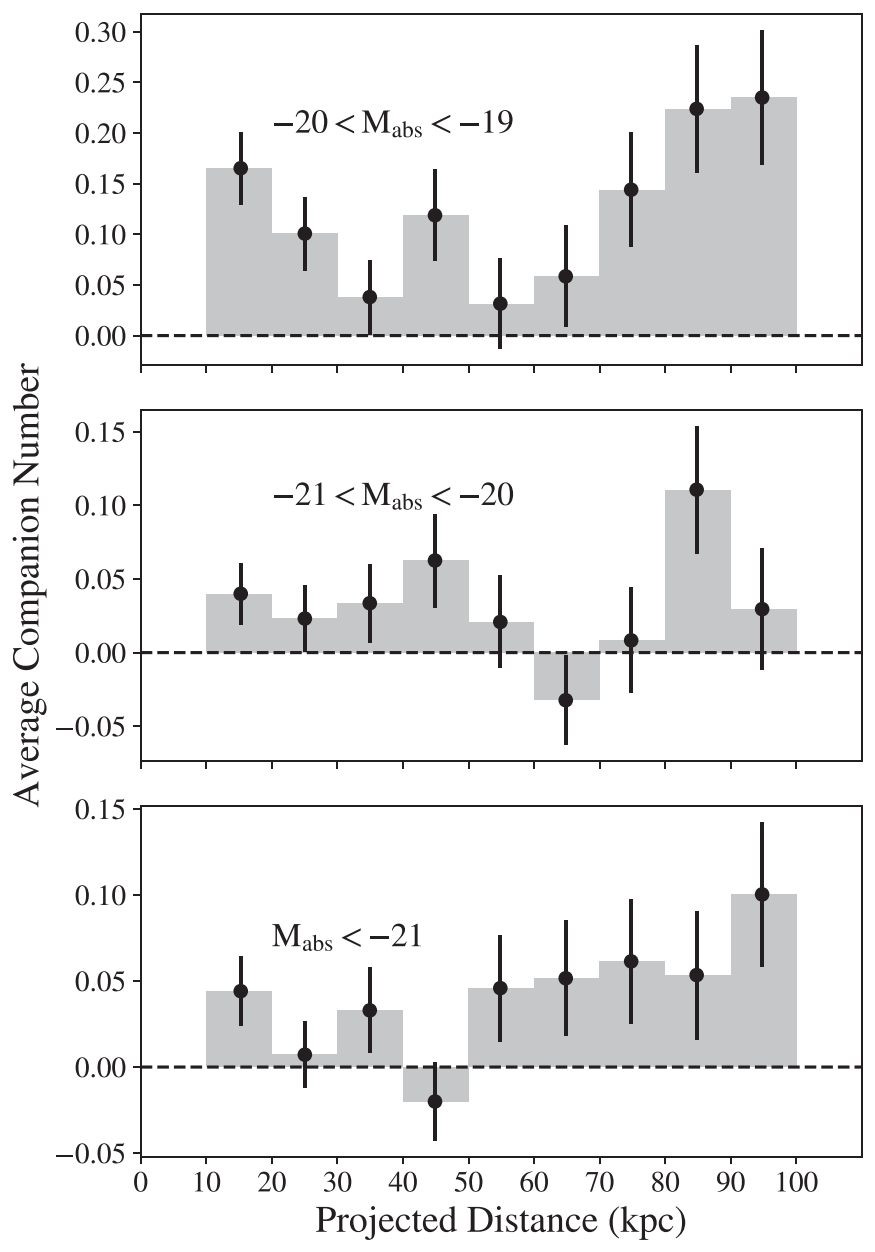

Figure 4. The average companion numbers of Sample A quasars. Each data point corresponds to a distance bin of $\Delta d=10 \mathrm{kpc}$. All the error bars represent $1 \sigma$ error assuming a Poisson distribution for the number of companions (same for all the other figures).

\section{Comparing the Companion Fraction of Quasars with a Galaxy Control Sample}

To compare the companion distribution of quasar host galaxies and normal galaxies, we use the 3D-HST galaxy catalog (Brammer et al. 2012; Skelton et al. 2014; Momcheva et al. 2016) to construct a control sample of galaxies. The 3D$H S T$ is an HST Treasury program to provide ACS and WFC3 images and grism spectroscopy over five fields: the Cosmic Evolution Survey (COSMOS), the Great Observatories Origins Deep Survey (GOODS), including GOODS-north and GOODSsouth, the All-wavelength Extended Groth Strip International Survey (AEGIS), and the Ultra Deep Survey (UDS) with a combined usable area of $\sim 0.25 \mathrm{deg}^{2}$. In this work, we use the 3D-HST photometry catalog v4.1.5, which is the latest version. It provides ACS and WFC3/IR broadband fluxes of galaxies, including F160W, F140W, and F125W for WFC3/IR images, as well as F814W and F606W for ACS images. The catalog also contains stellar mass and photometric redshifts of galaxies, derived with the methods described in Skelton et al. (2014).

Quasar host galaxies are believed to be massive $\left(M_{*} \gtrsim 10^{10} M_{\odot}\right.$, e.g., Dunlop et al. 2003; Matsuoka et al. 2014). We thus construct a "massive galaxy sample" by selecting all the galaxies that have $M_{*}>10^{10} M_{\odot}$ and photometry flag USE_PHOT $=1$ (which means good photometry) in the $3 \mathrm{D}-H S T$ photometry catalog. The control sample 
galaxies are randomly drawn from the massive galaxy sample and share the same redshift distribution as our quasars. This is done by evenly dividing the redshift range $(0.3<z<3)$ into nine bins and randomly selecting galaxies in each redshift bin, so that the fraction of galaxies in a specific redshift bin among all the control sample galaxies equals to the fraction of quasars in that redshift bin among all the quasars.

All projected companions of the control sample galaxies with a projected distance $10 \mathrm{kpc}<d<100 \mathrm{kpc}$ are selected in the same way as quasars. Since the 3D-HST contains five fields with different depths, the "background object density" of a control sample galaxy is approximated by the object surface number density of the 3D-HST field where the galaxy is located. We use F814W magnitudes to make magnitude cuts when counting companions, since F814W images dominate our quasar sample (Figure 2).

\subsection{Making Absolute Magnitude Cuts Based on Stellar Mass}

In Section 3.3, we show the number of companions around quasars as a function of companion flux, where the flux of companions are described by absolute magnitudes without $K$ corrections. This result is not suitable for studying the redshift evolution of quasar companions or comparing quasars with control sample galaxies, since different bands are used in the analysis of the quasars (all the eight broad bands of ACS/WFC) and the galaxies (F814W only). It is difficult to perform $K$ corrections in this work, because most of the quasars have only been observed in one band. Therefore, we use an abundance matching technique to convert stellar mass cuts to magnitude cuts in each band, which allows us to set magnitude cuts consistently at any redshift in all the eight bands. In short, for a given stellar mass $M_{*}$, we find a magnitude (denoted by $m\left(M_{*}\right)$ ) such that that the number of galaxies that are more massive than $M_{*}$ (denoted by $N\left(M_{*}\right)$ ) is the same as the number of galaxies that are brighter than $m\left(M_{*}\right)$. We describe the detailed procedures below.

The analysis is based on the photometric catalog of the UDS field in the 3D-HST project. The UDS photometric catalog is used because it contains the Johnson $B, V, R$ and SDSS $i^{\prime}, z^{\prime}$ photometry, which can be converted to $H S T$ broadband magnitudes and be compared with the quasars. The conversion is done as follows. The SDSS $i^{\prime}, z^{\prime}$ magnitudes are converted to Johnson I magnitude according to Jordi et al. (2006), then the $B V R I$ magnitudes are converted to ACS/WFC broadband magnitudes according to Sirianni et al. (2005). The conversion from BVRI to F850LP was not provided in Sirianni et al. (2005), so we used SDSS $z^{\prime}$ as an approximation of the F850LP magnitudes. We estimate the difference between SDSS $z^{\prime}$ and ACS/WFC F850LP magnitudes using the Exposure Time Calculator for HST ACS/WFC using typical galaxy templates. The difference is smaller than 0.02 mag. We select galaxies in the UDS photometric catalog with USE_PHOT $=1$ to construct the galaxy sample for the abundance matching technique. Note that this galaxy sample is different from the control sample; we only use the UDS field in the five 3D-HST fields and do not set any stellar mass cut on this sample.

For a quasar at redshift $z_{q}$, the magnitude cut (in an arbitrary band) corresponding to a stellar mass $M_{*}$ is estimated as follows. First, all objects with photometric redshift $\left(z_{\text {phot }}\right)$ that satisfy $z_{q}-0.1<z_{\text {phot }}<z_{q}+0.1$ in the UDS catalog are selected, constructing a "magnitude-cut-setting galaxy sample." Typically the redshift-matched galaxy sample contains $1000 \sim 3000$ galaxies. The magnitudes of the objects in the

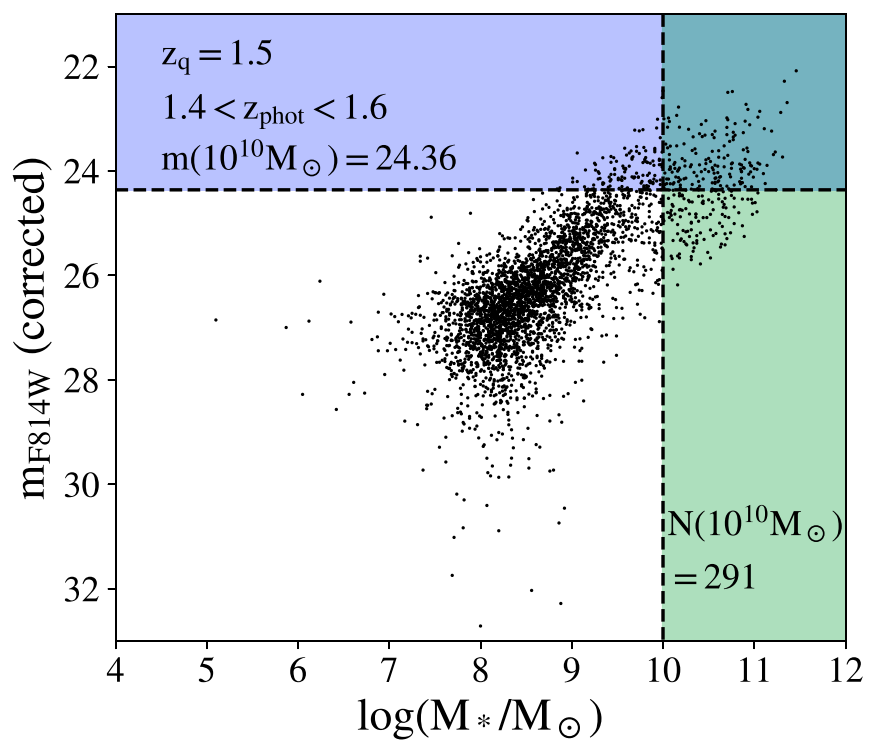

Figure 5. An example of converting stellar mass limits into apparent magnitude limits. In this example, we convert a stellar mass of $10^{10} M_{\odot}$ to F814W magnitude for $z \sim 1.5$ objects. We first select objects in the UDS catalog with photometric redshift $1.4<z_{\text {phot }}<1.6$, and correct their F814W magnitude according to Equation (3). There are 291 objects with $M_{*}>10^{10} M_{\odot}$ (objects in the green shade), which is also the number of objects with corrected F184W magnitude brighter than $m\left(10{ }^{10} M_{\odot}\right)=24.36$ (objects in the blue shade).

magnitude-cut-setting galaxy sample are corrected to $z_{q}$ according to the photometric redshifts, i.e., given a galaxy with a photometric redshift $z_{\text {phot }}$ and an apparent magnitude $m_{\text {raw }}$, we calculate the corrected magnitude by

$$
m=m_{\text {raw }}+5 \log \left[D_{L}\left(z_{q}\right) / D_{L}\left(z_{\text {phot }}\right)\right],
$$

where $D_{L}(z)$ is the luminosity distance. We then count the number of objects with stellar masses larger than the given value $M_{*}$ (referred to as $N\left(M_{*}\right)$ ) in the magnitude-cut-setting galaxy sample. The value of $N\left(M_{*}\right)$ varies from several hundred (for $M_{*}=10^{9} M_{\odot}$ ) to about 20 (for $M_{*}=10^{11} M_{\odot}$ ). Finally, we find the magnitude limit $m\left(M_{*}\right)$ so that there are $N\left(M_{*}\right)$ objects that are brighter than $m\left(M_{*}\right)$.

As an example, Figure 5 illustrates the process of estimating $m\left(10^{10} M_{\odot}\right)$ in $\mathrm{F} 814 \mathrm{~W}$ band at $z=1.5$. The number of objects in the green and the blue shade is equal. By definition, we can estimate the number of quasar companions with stellar masses larger than $M_{*}$ by counting objects that are brighter than $m\left(M_{*}\right)$.

For each quasar image, we set up three magnitude limits, $m\left(10^{9} M_{\odot}\right), m\left(10^{10} M_{\odot}\right)$, and $m\left(10^{11} M_{\odot}\right)$. When comparing quasars with the galaxy control samples, we will discuss companions with $m\left(10^{9} M_{\odot}\right)>m>m\left(10^{10} M_{\odot}\right)$ (faint), $m\left(10^{10} M_{\odot}\right)>m>$ $m\left(10^{11} M_{\odot}\right)$ (intermediate), and $m<m\left(10^{11} M_{\odot}\right)$ (bright). Correspondingly, we use Sample B throughout Section 4. Since most quasar host galaxies have stellar mass $M_{*}>10^{10} M_{\odot}$, "intermediate" companions are mainly associated with major mergers (mass ratio is close to 1), while "faint" companions are mainly related to minor mergers (mass ratio is larger than 3 ). The case of "bright" companions is more complicated; these systems might be associated with minor mergers where the quasar host galaxy is the less massive progenitor, or major mergers if the quasar host galaxy is as massive as $10^{11} M_{\odot}$. Studies on quasar host galaxies (e.g., 


\section{Sample B}

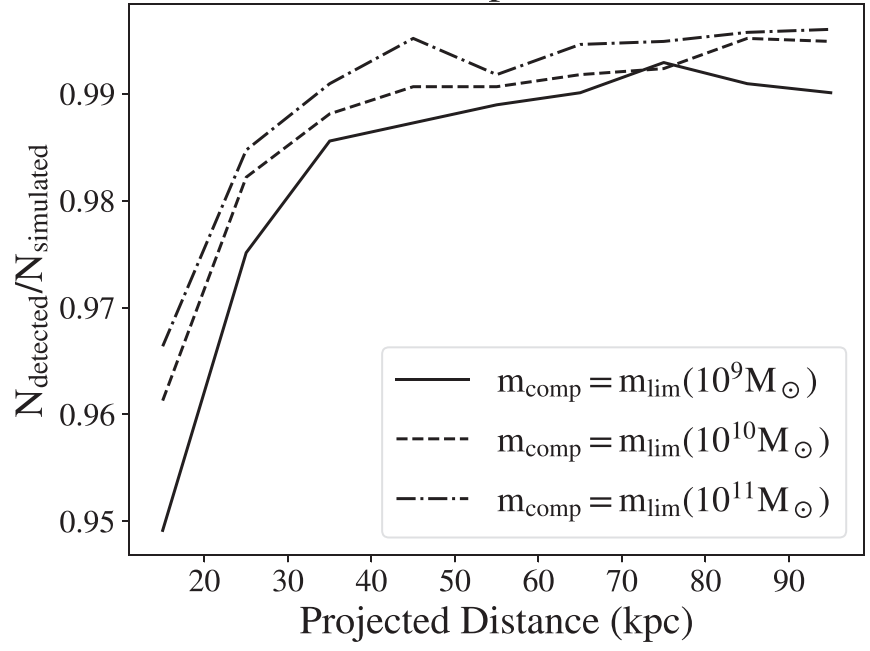

Figure 6. The detected fraction of quasar companions that have magnitudes of $m\left(10^{9} M_{\odot}\right), m\left(10^{10} M_{\odot}\right)$, and $m\left(10^{11} M_{\odot}\right)$, estimated by simulated images. The detected fraction is higher than $95 \%$ for all the companions of interest.

Matsuoka et al. 2014; Yue et al. 2018) show that only a small fraction of quasar host galaxies have stellar masses larger than $10^{11} M_{\odot}$, thus most systems with bright companions should be related to minor mergers. We estimate the completeness of companion detection for companions with $m\left(10^{9} M_{\odot}\right), m\left(10^{10} M_{\odot}\right)$, and $m\left(10^{11} M_{\odot}\right)$, using the same method as described in Section 3.2. The result is presented in Figure 6, which shows that the completeness of companion detection is higher than $95 \%$ in the simulated images.

Similar magnitude limits are calculated for galaxies in the control sample using the $\mathrm{F} 814 \mathrm{~W}$ magnitude. At $z=3$, the faintest companions that we consider in this study have magnitude $m_{\mathrm{F} 814 W}\left(10^{9} M_{\odot}\right)=26.3$. At this magnitude, about $2 \%$ objects in the 3D-HST catalog have signal-to-noise ratios smaller than 3, and the completeness of companion counting will drop toward fainter magnitudes (and thus higher redshift). This is the reason why our sample only contains $z<3$ objects.

\subsection{Comparison between Companions around Quasars and Galaxies}

We calculate the number of faint, intermediate, and bright companions of quasars in Sample B. Figure 7 shows the average number of physical companions, both for quasars and the control sample galaxies. Quasars have fewer intermediate companions at $d<60 \mathrm{kpc}$. At larger distances, the number of companions around quasars and galaxies are roughly the same. No significant difference can be seen for faint and bright companions between quasars and galaxies.

For Sample B quasars, the average numbers of "close companions" (companions with a project distance to quasars of $10 \mathrm{kpc}<d<30 \mathrm{kpc})$ are

$$
\begin{aligned}
& \bar{N}_{\text {comp }, \mathrm{Q}}(\text { faint })=0.233 \pm 0.043 \\
& \bar{N}_{\text {comp }, \mathrm{Q}}(\text { intermediate })=0.004 \pm 0.021 \\
& \bar{N}_{\text {comp }, \mathrm{Q}}(\text { bright })=0.012 \pm 0.018,
\end{aligned}
$$

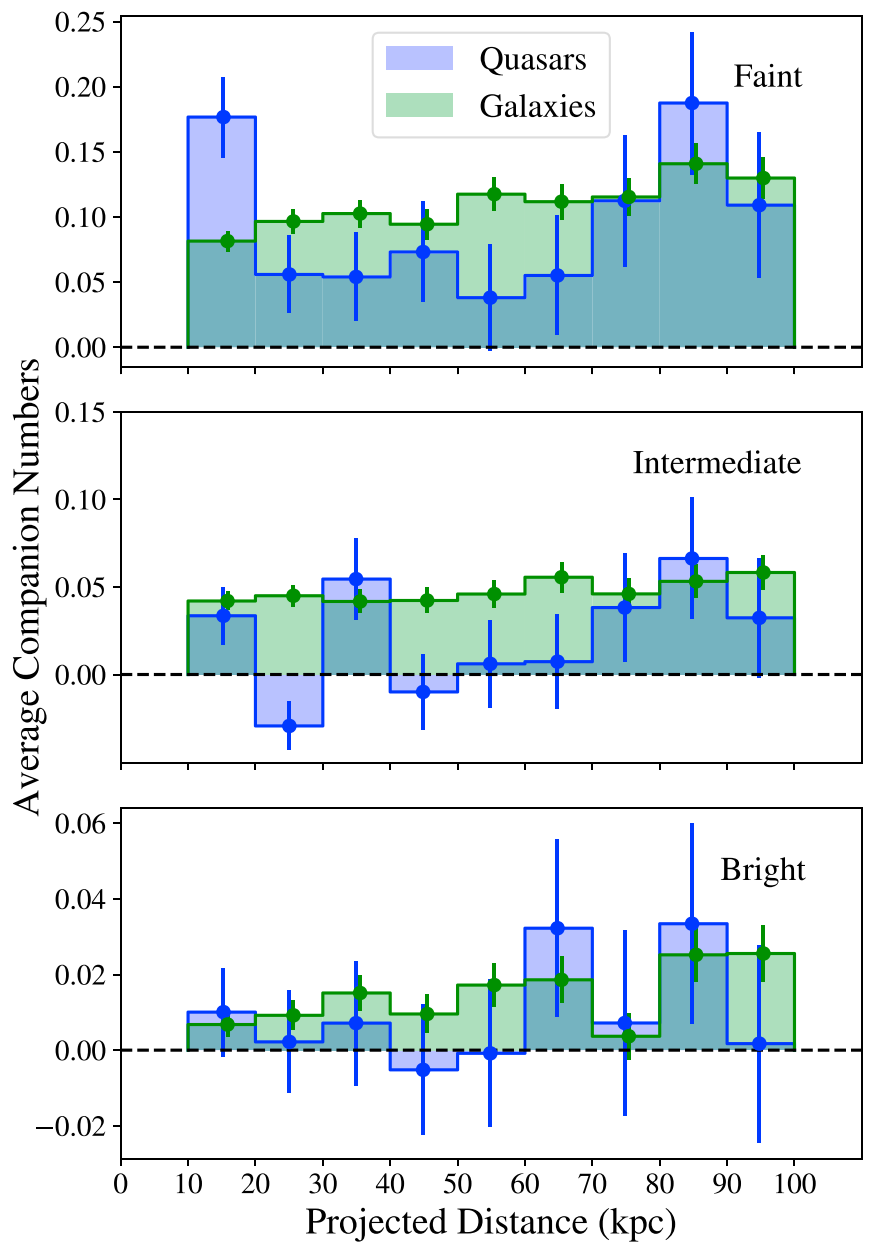

Figure 7. The average number of companions of quasars and the control sample galaxies. Each data point corresponds to a distance bin of $\Delta d=10 \mathrm{kpc}$. Small $x$-axis offsets are added to the error bars to make them distinguishable.

and the comparison galaxy sample has

$$
\begin{aligned}
& \bar{N}_{\text {comp }, \mathrm{G}}(\text { faint })=0.178 \pm 0.012 \\
& \bar{N}_{\text {comp,G }}(\text { intermediate })=0.087 \pm 0.008 \\
& \bar{N}_{\text {comp,G }}(\text { bright })=0.016 \pm 0.005 .
\end{aligned}
$$

Quasars show a 3.7 $\sigma$ deficit of close intermediate companions, and have numbers of faint and bright companions similar to those of normal galaxies.

Figure 8 shows the average number of close companions of quasars and control sample galaxies as a function of redshift. The average number of companions around control sample galaxies increases toward high redshift, both for faint and bright companions. This result is expected since the universe is more crowded at high redshift. Meanwhile, the average number of companions around quasars does not significantly evolve with redshift.

Figure 9 shows the average number of close companions as a function of quasar luminosity. More luminous quasars have more faint companions, while the number of intermediate companions decreases with quasar luminosity. Given the statistical error, both trends are not significant. The number of bright companions does not show a luminosity dependence. 

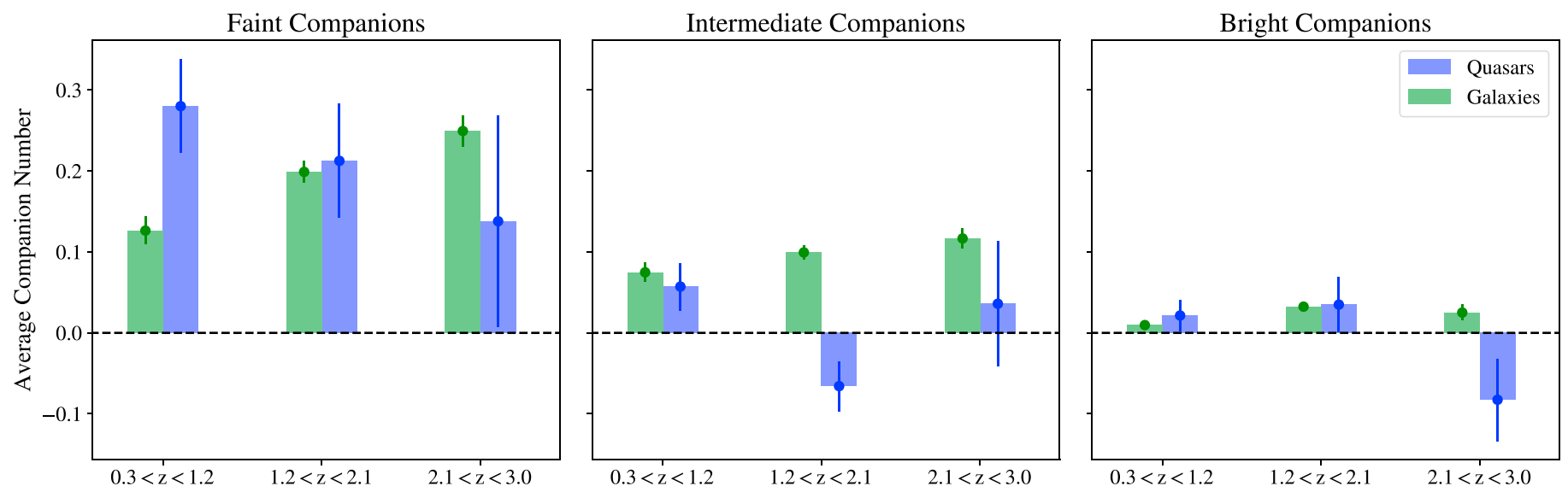

Figure 8. The redshift evolution of average number of companions around quasars. The average number of companions of galaxies in the control sample is also included.

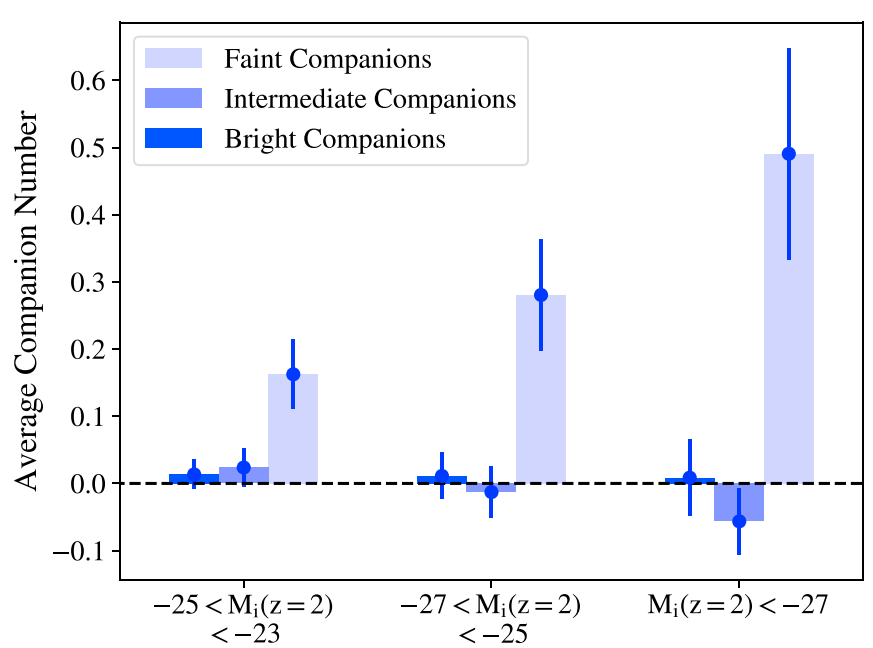

Figure 9. The evolution of the average number of companions around quasars with quasar luminosity.

We also examine the relationship between average companion number of quasars and other quasar properties, including:

1. Broad absorption line (BAL) features. The SDSS quasar catalogs contain BAL flags, while the Véron catalog does not have such information, thus our BAL and non-BAL quasar sample only contain SDSS quasars. We find that BAL quasars and non-BAL quasars have consistent numbers of companions (both faint and intermediate) at a $1 \sigma$ level.

2. Radio loudness. We match our quasar catalog with the Faint Images of the Radio Sky at Twenty-Centimeters (FIRST; e.g., Becker et al. 1995; White et al. 1997) survey catalog. We divide the quasars that were covered by the FIRST survey into two subsamples, namely, "radio-loud (RL) quasars" and "radio-quiet (RQ) quasars," according to whether they were detected by the FIRST survey. We find that RL quasars tend to have more "faint" companions than RQ quasars. The average number of "faint" companions is $0.523 \pm 0.144$ around RL quasars and $0.153 \pm 0.047$ around RQ quasars, which is a $2.4 \sigma$ difference. The two samples have similar numbers of intermediate companions (consistent at a $1 \sigma$ level).
3. IR brightness. We match our quasar catalog with the Wide-field Infrared Survey Explorer (WISE; e.g., Wright et al. 2010) ALLWISE source catalog. Since WISE W3 and $W 4$ are usually not deep enough for faint quasars, we use the WISE W1 and W2 fluxes to estimate the rest frame $2 \mu \mathrm{m}$ magnitude $\left(M_{2 \mu}\right)$ assuming that the near-infrared SED of quasars can be represented by a power law. We then evenly divide the quasars into two subsamples according to their $M_{2 \mu}-M_{i}(z=2)$ color, referred to as "IR-bright quasars" and "IR-faint quasars," respectively. One potential problem is that the near-infrared SED of some quasars cannot be well fitted by a single power law (e.g., Glikman et al. 2006; Hernán-Caballero et al. 2016). To investigate the influence of fitting quasar SEDs by a single power law, we use the quasar spectrum template in Hernán-Caballero et al. (2016) to fit the WISE W1 and W2 fluxes of the quasars and define the two subsamples based on the template-estimated rest frame $2 \mu \mathrm{m}$ magnitude. Among all the IR-bright (IR-faint) quasars defined using the power-law fit, $11.6 \%$ are classified as IR-faint (IRbright) in the template-based classification. In both cases, IR-bright and IR-faint quasars have similar numbers of faint and intermediate companions (consistent at a $1.5 \sigma$ level).

\section{Discussion}

\subsection{Selection Effects}

Our sample consists of quasars from the SDSS quasar catalogs and the Véron quasar catalog that have been observed by HST ACS/WFC broadband imaging. The parent sample (SDSS + Véron) includes almost all quasars known to date. Selection effects may be introduced by selecting quasars that have ACS/WFC imaging. Among the 532 quasars in our sample, 402 of them $(76 \%)$ were observed by ACS/WFC for purposes that were irrelevant to AGN science (e.g., photometric surveys, studies on supernovae or local galaxies). Using the subsample of quasars which were observed in AGN-unrelated programs, we estimate the average companion numbers around quasars to be $0.194 \pm 0.052,0.003 \pm 0.028$, and $0.005 \pm$ 0.023 for faint, intermediate, and bright companions. These numbers are close to the result we get using the whole sample. For the rest of the sample, the goal of the original HST program was related to AGN science, which could introduce 
complicated selection effect, e.g., for the programs that targeted a specific class of AGN that could have higher merger fraction.

Our results described in Section 4.2 are based on several assumptions, which may also introduce systematic errors. The main assumption we make is that the companion fluxes can be converted to stellar masses as described in Section 4.1. This cannot be applied to individual objects. However, if quasar companions follow the same stellar mass and flux distribution as normal galaxies, our method will provide the correct numbers of companions that fall in a certain stellar mass range. This method ignores the possible influence of quasars on their companions, which is a complicated effect and is difficult to correct. When applying the results, this potential systematic error must be kept in mind. On the other hand, the "primary results" in Section 3.3 are free from this systematic error.

\subsection{The Fraction of Merger-triggered Quasars}

Our results suggest that there is a deficit of companions around quasars compared with inactive galaxies. We interpret the difference as a result of the difference between the merging history of quasars (especially merger-triggered ones) and normal galaxies. Here we first review the merger-triggering model of quasars briefly and discuss how many companions would we expect around a merger-triggered quasar.

According to simulations (e.g., Lotz et al. 2010), the evolution of a galaxy merger will experience five stages: the first encounter, the largest separation, the second encounter, the final coalesce, and the post-merger remnant. Each stage lasts for $\lesssim 0.5 \mathrm{Gyr}$. The typical lifetime of a quasar is $10 \sim 100$ Myr (e.g., Hopkins et al. 2008), which means that the morphological properties of quasar host galaxies will not change significantly during the life of the quasar.

Previous simulations on galaxy major mergers triggering quasars (e.g., Di Matteo et al. 2005; Springel et al. 2005; Hopkins et al. 2006; Newton \& Kay 2013) have suggested that quasars emerge at the final coalesce stage. This can be understood in the following picture. In the merger-triggering model, the quasar activity emerges when strong gas inflows feed the SMBH. This process requires the gas content to be highly disturbed. Simulations of galaxy major mergers show that the disturbed features are the most prominent at the second encounter stage. It takes some time for the gas to reach and feed the $\mathrm{SMBH}$, thus strong quasar activities are expected to emerge at the final coalesce. As an observational evidence, Ellison et al. (2013) found that post-mergers in SDSS have a high AGN fraction.

With some simple calculations, we derive the fraction of merger-triggered quasars using the number of close companions. Assuming that quasars are either triggered by secular evolution or mergers, and the fraction of merger-triggered quasar is $\alpha$. We use $\bar{N}_{\text {comp,QS }}$ and $\bar{N}_{\text {comp,QM }}$ to denote their average number of companions, where "Q" means "quasar," "S" stands for "secular evolution" and "M" means "merger." The observed average companion number of quasars should be $\bar{N}_{\text {comp,Q }}=(1-\alpha) \bar{N}_{\text {comp,QS }}+\alpha \bar{N}_{\text {comp,QM }}$, from which we have

$$
\alpha=\frac{\bar{N}_{\text {comp,QS }}-\bar{N}_{\text {comp,Q }}}{\bar{N}_{\text {comp,QS }}-\bar{N}_{\text {comp,QM }}} \text {. }
$$

We further assume that (1) secular-evolution-triggered quasars have the same number of companions as normal galaxies ( $\bar{N}_{\text {comp,QS }} \approx \bar{N}_{\text {comp,G }}$, where " $\mathrm{G}$ " means inactive galaxies), since the secular evolution will not influence the merging process; and (2) merger-triggered quasars have no close companions $\left(\bar{N}_{\text {comp, QM }} \approx 0\right)$, since the two progenitor galaxies of the merger-triggered quasar have already merged into a single galaxy. We then have

$$
\alpha=\frac{\bar{N}_{\text {comp }, \mathrm{G}}-\bar{N}_{\mathrm{comp}, \mathrm{Q}}}{\bar{N}_{\mathrm{comp}, \mathrm{G}}} .
$$

Figure 7 indicates that the difference between the average number of companions of quasars and galaxies varies with the companion stellar mass. As a result, $\alpha$ depends on the companion mass. This result is not surprising since we expect that major mergers and minor mergers have different probabilities to trigger quasars. We can interpret $\alpha\left(M_{\text {companion }}\right)$ as the fraction of quasars that are triggered by a merger where one progenitor galaxy had a stellar mass of $M_{\text {companion. }}$ Equations (4) and (5) describe the average number of companions of our sample. According to Equation (7), we have $\alpha$ (faint $)=-0.31 \pm 0.26, \alpha($ intermediate $)=0.95 \pm 0.25$ and $\alpha$ (bright $)=0.23 \pm 1.14$. Since intermediate and faint companions are associated with major and minor mergers, respectively, our toy model indicates that there should be a significant fraction of quasars that are triggered by major mergers, and that minor mergers have a small contribution in quasar triggering. The large error for the bright companions does not allow for a strong conclusion.

We now discuss the impact of our assumptions in the analysis above. The first assumption is that secular-evolutiontriggered quasars have the same number of companions as normal galaxies. Close neighbors of galaxies can disturb their gas kinematics and lead to gas inflows. However, gas inflows generated in this way are usually not strong enough to feed a quasar, thus the environmental influence should be minor. Previous studies have also suggested that secular evolution is only responsible for faint AGNs (e.g., Treister et al. 2012). Moreover, according to Equation (6), a larger number of close companions around secular-evolution-triggered quasars will lead to a higher fraction of merger-triggered quasars. The second assumption is that there are no companions around merger-triggered quasars. This assumption is clearly oversimplified. Our main idea is that, in the picture of a mergertriggered quasar discussed above, the quasar host galaxy is a galaxy merger that has entered the final coalesce stage, and we can only see one galaxy rather than a pair. Two possible errors come into this assumption. On the one hand, a merger-triggered quasar may not show up exactly in the final coalesce stage. It is possible that the quasar is triggered earlier when the two merging galaxies are still distinguishable. We suggest that the influence of this possible error should be small, however, because we only count companions with distance larger than $10 \mathrm{kpc}$, and most observed merging galaxy pairs are closer. On the other hand, if there are nearby galaxies that are not involved in this merging event, we will have a non-zero companion number for merger-triggered quasars. Given the distance cut we applied $(10 \mathrm{kpc}<d<30 \mathrm{kpc})$, such cases should be rare. In both cases, Equation (6) suggests that, if $\bar{N}_{\text {comp, QM }}>0$, the fraction of merger-triggered quasars will become even larger, which will further strengthen our conclusion. 


\subsection{A Unified Picture of AGN Triggering}

Conclusions from previous studies on AGN merger fractions have been ambiguous. There are both results indicating enhanced merging fractions of AGN (e.g., Ellison et al. 2011; Silverman et al. 2011; Satyapal et al. 2014; Fan et al. 2016; Goulding et al. 2018; Weston et al. 2017) and indicating no difference between AGN and normal galaxies (e.g., Cisternas et al. 2011; Schawinski et al. 2011; Kocevski et al. 2012; Villforth et al. 2017). Our result suggests that there should be a significant fraction of major-merger-triggered quasars. We consider several possible reasons for this ambiguity.

First, most of these studies used distinct AGN samples. The AGN samples vary from emission-line-ratio selected (e.g., Ellison et al. 2011), near-IR selected (e.g., Satyapal et al. 2014; Fan et al. 2016; Goulding et al. 2018; Weston et al. 2017), X-ray selected (e.g., Cisternas et al. 2011; Silverman et al. 2011; Kocevski et al. 2012; Villforth et al. 2017), to optical selected (this work). We notice that all the studies using nearIR-selected AGN samples reach a conclusion that AGN have an enhanced merging fraction, while most of the $\mathrm{X}$-ray selected AGN samples do not show a significant difference from inactive galaxies. This result can be explained if different populations of AGN emerge in different stages of galaxy mergers. The luminosities of the AGN samples are also different, which is believed to have a crucial influence on AGN merging fraction. Previous observations using optical data focused mainly on low-luminosity AGNs to avoid the strong emission from the central nuclei. Most studies on highluminosity AGNs are based on either X-ray or infrared data (for obscured ones). Comparing to these studies, our sample consists of optical-selected AGNs and spans a wide range of luminosity $\left(-31<M_{i}(z=2)<-23\right)$.

Second, the method of these studies might introduce some biases. Most of the previous studies used disturbed features in quasar host galaxies as indicators of recent merger events. There have been arguments that these features should be able to survive until the quasar activity emerges (e.g., Cisternas et al. 2011; Villforth et al. 2017). However, this depends on the model of galaxy mergers and AGN triggering, which is highly uncertain and varies from object to object. Even if the average timescale of disturbed features may be long enough, it is still possible to miss some mergers and thus underestimate the merger fraction of AGN. The uncertainty in identifying disturbed features in AGN host galaxies might be more severe for bright unobscured AGNs that need PSF subtraction, given the difficulty of PSF modeling.

It is also difficult to distinguish major mergers and minor mergers based on the host galaxy morphology. If minor mergers do not contribute to the triggering of quasars (as indicated by our result), including them as "recent merging systems" will increase the number of identified merging systems and introduce extra uncertainties when testing the major-merger-triggering mechanism.

In comparison, we identify mergers by counting companions. This will not introduce significant biases against the latestage mergers, where the disturbed features in the galaxies might have already faded away. It is also more straightforward to distinguish major and minor mergers since we can estimate the companion mass. Counting companions is an accessible way for nearly all populations of AGN, and does not require superb angular resolution, which makes it easier to build a large, unbiased sample.

Keeping the possible biases in mind, we find that most of the results are consistent with the picture where:

(1) Mergers are the main triggering mechanism for highluminosity AGNs, and secular evolution is mainly responsible for low-luminosity AGNs. There have been simulations claiming that secular evolution is not powerful enough to trigger the most luminous quasars (e.g., Treister et al. 2012). Previous observations have also reported that the merger fraction increases with AGN luminosity (e.g., Fan et al. 2016). We find that the average number of intermediate companions decreases with quasar luminosity. According to Figure 9 and Equation (7), our result indicates that luminous quasars are more likely to be triggered by major mergers.

(2) Merger-triggered AGNs evolve from an obscured to an unobscured phase. The transition happens when the radiation and material outflows blow away the dust around the active nucleus. Consequently, IR-selected AGNs (which are dustier) might represent an earlier stage of AGN evolution, and it is easier to detect the disturbed features in their host galaxies than other populations of AGN. This picture is supported by simulations (e.g., Di Matteo et al. 2005; Hopkins et al. 2008), and can explain the discrepancy with previous observations, which reported that IR-selected AGNs have a larger merging fraction than X-ray-selected AGNs.

\subsubsection{Merger Rate of AGN versus AGN Rate in Mergers}

In comparison to works such as ours, which measures the fraction of mergers in quasars, some studies instead compared the fraction of AGN in merging and non-merging systems, quantified by the "AGN fraction ratio":

$$
R=\frac{P(\text { AGN } \mid \text { merger })}{P(\text { AGN } \mid \text { non-merger })},
$$

where $P(\mathrm{AGN} \mid$ merger) $(P(\mathrm{AGN} \mid$ non-merger $))$ is the probability of finding an AGN in a (non-)merging system. Previous studies found that merging systems are more likely to host AGN (e.g., $R \sim 2-7$ in Goulding et al. 2018, $R \sim 5-17$ in Weston et al. 2017), and concluded that galaxy mergers are the dominant triggering mechanism of the AGN in their sample. Using Bayesian analysis, we can calculate the AGN fraction ratio using the merger ratio in $\mathrm{AGN}$, and thus compare our result directly with previous studies. According to Bayes' theorem,

$$
\begin{aligned}
P(\mathrm{AGN} \mid \text { merger }) & =\frac{P(\text { merger } \mid \mathrm{AGN}) P(\mathrm{AGN})}{P(\text { merger })} \\
P(\text { AGN } \mid \text { non-merger }) & =\frac{P(\text { non-merger } \mid \mathrm{AGN}) P(\mathrm{AGN})}{P(\text { non-merger })},
\end{aligned}
$$

thus

$$
\begin{aligned}
R & =\frac{P(\text { non-merger })}{P(\text { merger })} \times \frac{P(\text { merger } \mid \mathrm{AGN})}{P(\text { non-merger } \mid \mathrm{AGN})} \\
& =\frac{1-P(\text { merger })}{P(\text { merger })} \times \frac{P(\text { merger } \mid \mathrm{AGN})}{1-P(\text { merger } \mid \mathrm{AGN})},
\end{aligned}
$$

where we apply $P($ merger $)+P($ non-merger $)=1$. 
In our sample, the estimated major-merger-triggered quasar fraction is $\alpha=0.95 \pm 0.25$, with a $3 \sigma$ lower limit of 0.22 . We assume that the secular-evolution-triggered quasars have the same merger fraction as inactive galaxies (i.e., $P_{\mathrm{S}}($ merger $\mid \mathrm{AGN})=$ $P$ (merger) $)$. We also have $P_{\mathrm{M}}($ merger $\mid \mathrm{AGN})=1$ by definition. We adopt the merger fraction of inactive galaxies from Villforth et al. (2017), which gave $P($ merger $) \approx 0.2$. This gives

$$
\begin{aligned}
& P(\text { merger } \mid \mathrm{AGN})=\alpha P_{\mathrm{M}}(\text { merger } \mid \mathrm{AGN}) \\
& \quad+(1-\alpha) P_{\mathrm{S}}(\text { merger } \mid \mathrm{AGN}) \geqslant 0.37,
\end{aligned}
$$

and $R \geqslant 2.4$ according to Equation (10), which is consistent with previous studies.

\section{Summary}

We investigate the number of companions around quasars that have $M_{i}(z=2)<-23$ at $0.3<z<3$. Based on the SDSS quasar catalogs and the Véron quasar catalog, we construct a sample of 532 quasars that have been observed by HST ACS/ WFC, and use the archival images to find all the companions around these quasars with projected distance of $10 \mathrm{kpc}<$ $d<100 \mathrm{kpc}$. PSF subtraction is done for all the quasars to enhance the detectability of close companions, and the fraction of missed companions was estimated to be less than $10 \%$ even for the faintest companion at the smallest projected distance of interest (Sections 3.2 and 4.1). We use galaxies in the 3D-HST photometric catalog to construct a redshift-matched sample of massive inactive galaxies as our control sample. We define "faint," "intermediate," and "bright" companions such that faint and bright companions are associated with minor mergers, and intermediate companions correspond to major mergers. We calculate the average number of companions of quasars and inactive galaxies, and propose an explanation as to the difference between quasars and normal galaxies. Our main conclusions are:

1. Both quasars and inactive galaxies show excesses of companion surface densities in their neighborhoods. Quasars show a deficit of intermediate companions at projected distance $d \lesssim 60 \mathrm{kpc}$, and have numbers of faint/bright companions similar to normal galaxies. The average number of companions around quasars show little evolution with redshift, and do not show significant dependence on absorption features, radio loudness, and IR luminosity. More luminous quasars have more faint companions and fewer intermediate companions, though both trends are not significant. The number of bright companions does not evolve with quasar luminosity.

2. By assuming that merger-triggered quasars have no close companions and secular-evolution-triggered quasars have the same number of companions as inactive galaxies, the deficit of close companions around quasars indicates that a significant fraction of quasars are triggered by major mergers.

3. Most of the previous studies are consistent with the picture where the merger-triggered fraction increases with AGN luminosity, and merger-triggered AGN evolves from an obscured to an unobscured phase. The ambiguity of previous results may be a result of biases introduced by the samples and the methods.

Using close companions as identifiers of merging systems does not require superb angular resolution, which makes it possible to constrain the AGN merger fraction using groundbased imaging. Ground-based surveys like the Hyper SuprimeCam Survey (Aihara et al. 2018) and the Large Synoptic Survey Telescope (Dark Energy Science Collaboration 2012) have angular resolution that is good enough for companion counting, and can provide a large sample to decrease the statistical error. Future studies utilizing these surveys will provide a more accurate estimate on the fraction of merger-triggered AGN, and put better constraints on AGN triggering models.

We acknowledge the support from HST-AR-14312 grant from the Space Telescope Science Institute. We acknowledge the useful suggestions from George H. Rieke, Ann Zabludoff, Richard Green, and Peter S. Behroozi. We acknowledge the useful comments and suggestions from the referee.

Based on observations made with the NASA/ESA Hubble Space Telescope, and obtained from the Hubble Legacy Archive, which is a collaboration between the Space Telescope Science Institute (STScI/NASA), the Space Telescope European Coordinating Facility (ST-ECF/ESA), and the Canadian Astronomy Data Centre (CADC/NRC/CSA).

This work is based on observations taken by the 3D-HST Treasury Program (HST-GO-12177 and HST-GO-12328) with the NASA/ESA Hubble Space Telescope, which is operated by the Association of Universities for Research in Astronomy, Inc., under NASA contract NAS5-26555.

Facility: HST.

\section{Appendix Ongoing Merging Systems}

In this appendix, we present the images and information of candidates of ongoing merging systems with quasar activity mentioned in Section 3.3.1. We visually inspected all the quasars in the master sample and select systems that show either a pair of interacting galaxies or some disturbed features like tidal tails. Figure 10 shows the images of the ongoing merging systems. All the images are $100 \times 100 \mathrm{kpc}$ in size. The information of these objects can be found in Table 3 .

Table 3

\begin{tabular}{|c|c|c|c|c|}
\hline Quasar Name & R.A. & Decl. & Redshift & Feature \\
\hline SDSS J005009.81-003900.6 & 00:50:09.81 & $-00: 39: 00.6$ & 0.728 & $\overline{\text { Interacting galaxies }}$ \\
\hline SDSS J005916.10+153816.1 & 00:59:16.10 & $+15: 38: 16.1$ & 0.354 & Interacting galaxies \\
\hline SDSS J020258.94-002807.5 & 02:02:58.94 & $-00: 28: 07.5$ & 0.339 & Tidal tail \\
\hline SDSS J080908.13+461925.6 & 08:09:08.13 & $+46: 19: 25.6$ & 0.657 & Tidal tail \\
\hline SDSS J110556.18+031243.1 & $11: 05: 56.18$ & $+03: 12: 43.1$ & 0.353 & Interacting galaxies \\
\hline
\end{tabular}

Information of Ongoing Merging Systems

Note. The full table is also available in FITS format at https://github.com/yuemh/qso_companion.

(This table is available in its entirety in machine-readable form.) 

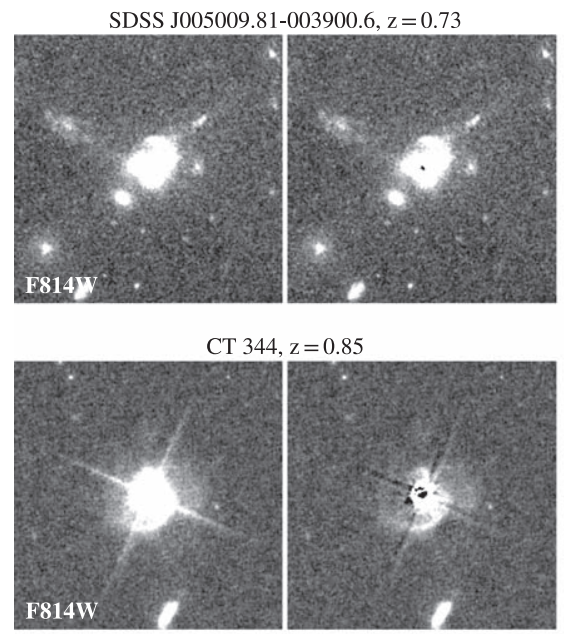

RXS J02282-4057, $\mathrm{z}=0.49$
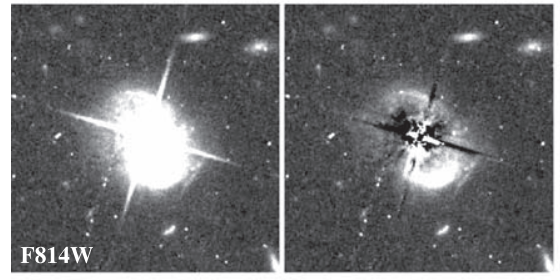

CDFS J03324-2741B, $\mathrm{z}=0.73$

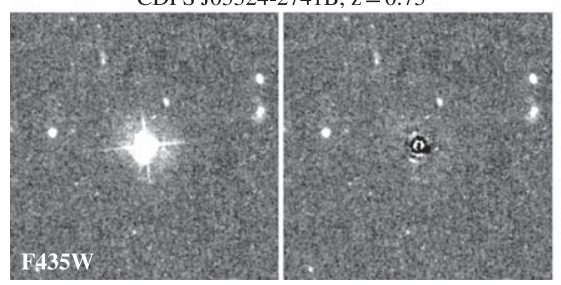

SDSS J005916.10+153816.1, $\mathrm{z}=0.35$

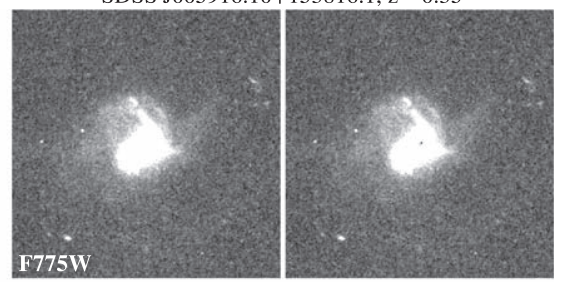

SDSS J020258.94-002807.5, $\mathrm{z}=0.34$
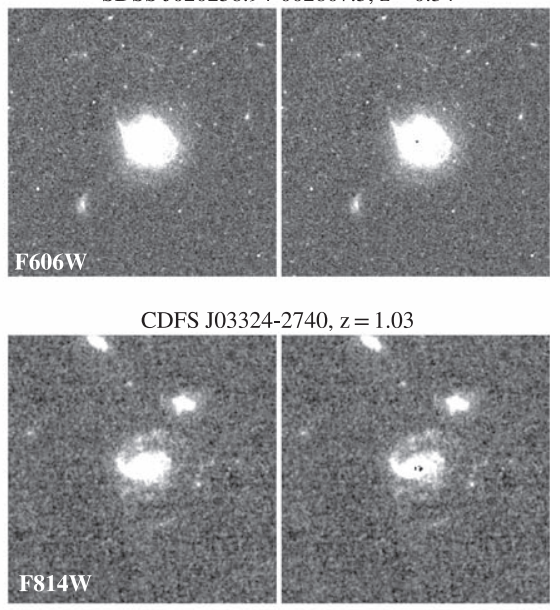

SDSS J080908.13+461925.6, $\mathrm{z}=0.66$

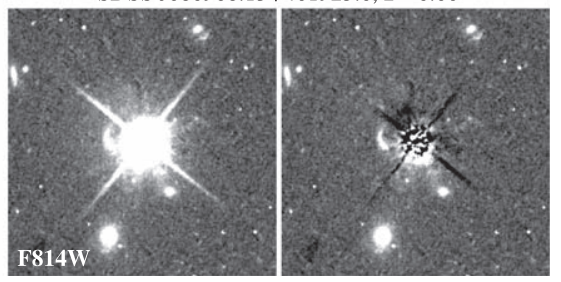

Figure 10. Images of ongoing merging systems in our master sample. The image sizes are $100 \times 100 \mathrm{kpc}$ at the redshift of the object. We present both the original image (left) and the PSF-subtracted image (right). 


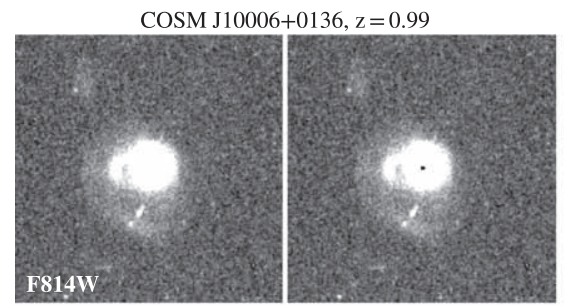

SDSS J110556.18+031243.1, $\mathrm{z}=0.35$

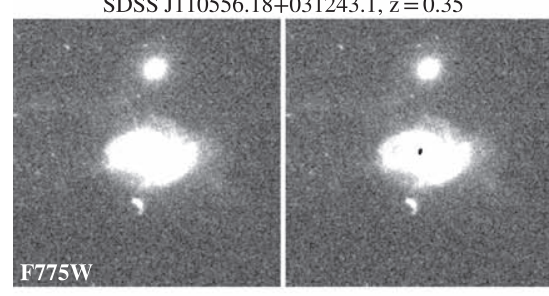

3C $263.0, z=0.65$

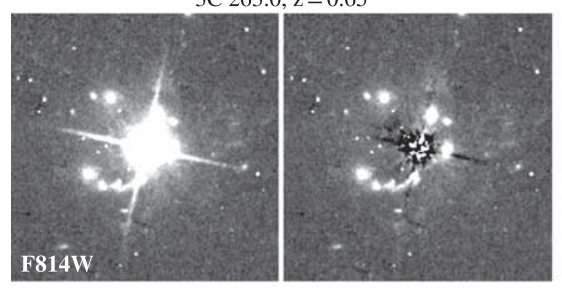

SDSS J115324.46+493108.7, $\mathrm{z}=0.33$

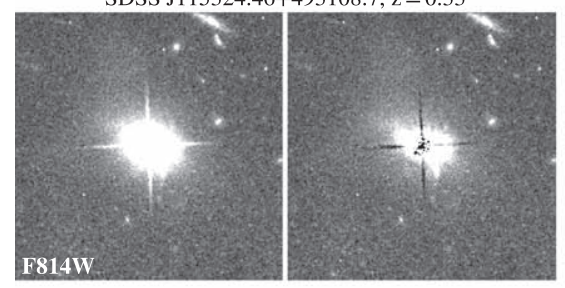

SDSS J155304.92+354828.6, $z=0.72$

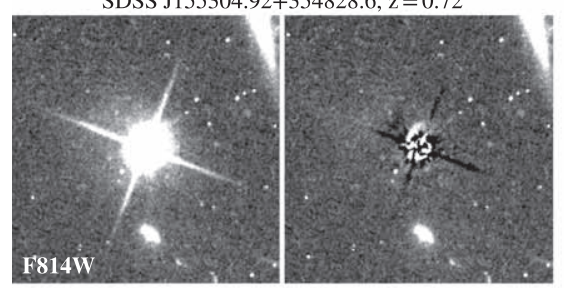

SDSS J170441.38+604430.5, z=0.37

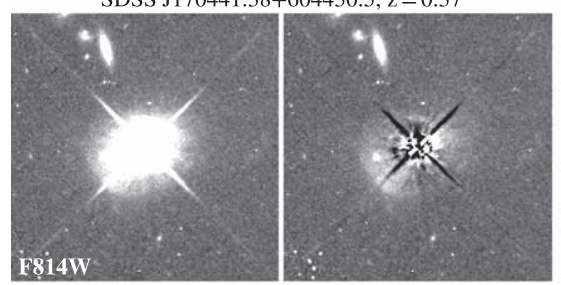

SDSS J172148.27+575805.3, $\mathrm{z}=1.62$

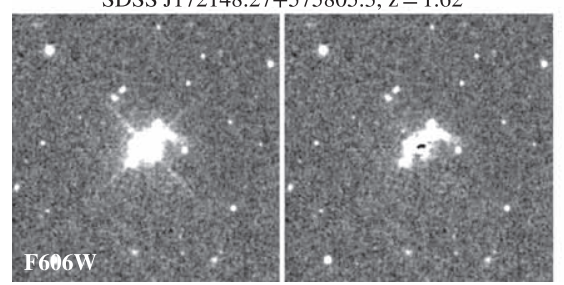

3C $249.1, z=0.31$

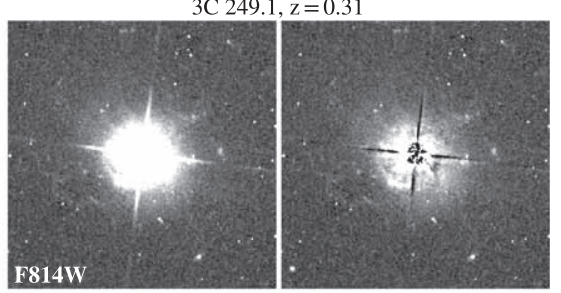

SDSS J113851.73+632955.6, $\mathrm{z}=2.62$

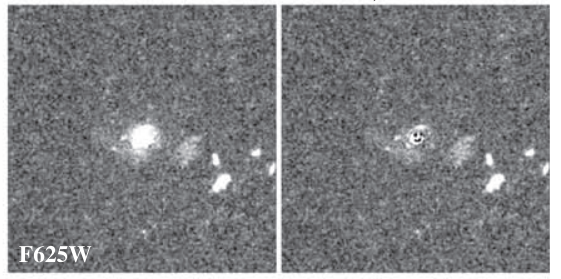

SDSS J114756.00-025023.4, $\mathrm{z}=2.56$

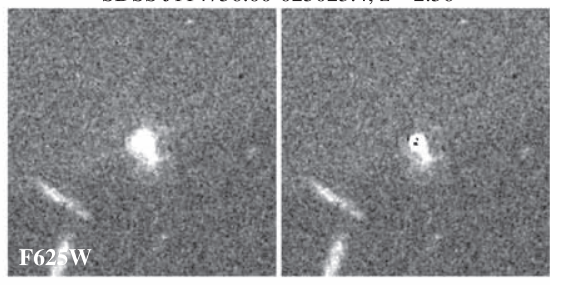

SDSS J141914.27+524608.6, $\mathrm{z}=2.70$

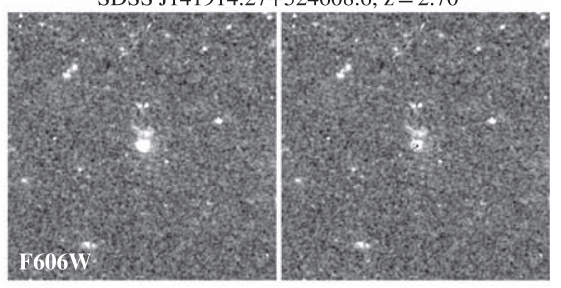

SDSS J164311.34+315618.4, $\mathrm{z}=0.59$

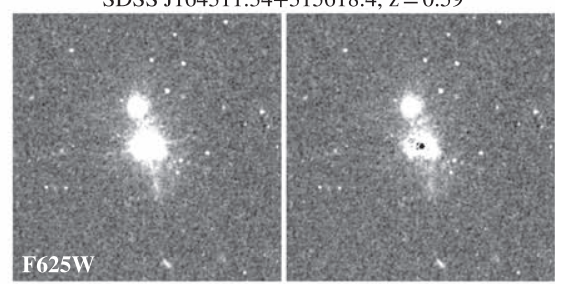

SDSS J171748.43+594820.6, $\mathrm{z}=0.76$

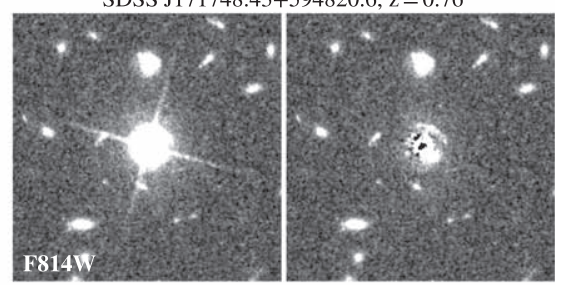

SDSS J173203.08+611751.8, $\mathrm{z}=0.36$

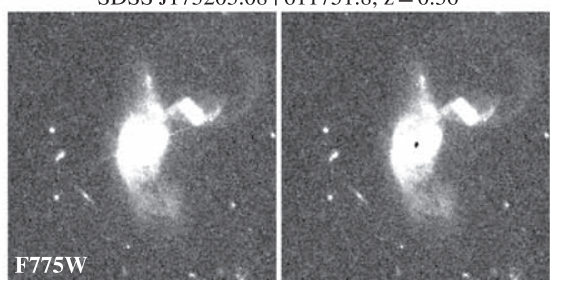

Figure 10. (Continued.) 


\section{ORCID iDs}

Minghao Yue (1) https://orcid.org/0000-0002-5367-8021

Xiaohui Fan (1) https://orcid.org/0000-0003-3310-0131

Jan-Torge Schindler (1) https://orcid.org/0000-0002-4544-8242

Ian D. McGreer (ib https://orcid.org/0000-0002-3461-5228

Yun-Hsin Huang (i) https://orcid.org/0000-0003-4955-5632

\section{References}

Aihara, H., Arimoto, N., Armstrong, R., et al. 2018, PASJ, 70, S4

Barnes, J. E., \& Hernquist, L. E. 1991, ApJL, 370, L65

Becker, R. H., White, R. L., \& Helfand, D. J. 1995, ApJ, 450, 559

Bertin, E., \& Arnouts, S. 1996, A\&AS, 117, 393

Brammer, G. B., van Dokkum, P. G., Franx, M., et al. 2012, ApJS, 200, 13

Cicone, C., Maiolino, R., Sturm, E., et al. 2014, A\&A, 562, A21

Cisternas, M., Jahnke, K., Inskip, K. J., et al. 2011, ApJ, 726, 57

Croton, D. J., Springel, V., White, S. D. M., et al. 2006, MNRAS, 365, 11

de Ravel, L., Le Fèvre, O., Tresse, L., et al. 2009, A\&A, 498, 379

Di Matteo, T., Springel, V., \& Hernquist, L. 2005, Natur, 433, 604

Dunlop, J. S., McLure, R. J., Kukula, M. J., et al. 2003, MNRAS, 340, 1095

Ellison, S. L., Mendel, J. T., Patton, D. R., \& Scudder, J. M. 2013, MNRAS, 435, 3627

Ellison, S. L., Patton, D. R., Mendel, J. T., \& Scudder, J. M. 2011, MNRAS, 418, 2043

Ellison, S. L., Patton, D. R., Simard, L., \& McConnachie, A. W. 2008, AJ, 135, 1877

Fan, L., Han, Y., Fang, G., et al. 2016, ApJL, 822, L32

Glikman, E., Helfand, D. J., \& White, R. L. 2006, ApJ, 640, 579

Gonzaga, S., et al. 2012, The DrizzlePac Handbook, HST Data Handbook

Goulding, A. D., Greene, J. E., Bezanson, R., et al. 2018, PASJ, 70, S37

Grogin, N. A., Conselice, C. J., Chatzichristou, E., et al. 2005, ApJL, 627, L97

Hernán-Caballero, A., Hatziminaoglou, E., Alonso-Herrero, A., et al. 2016, MNRAS, 463, 2064

Hopkins, P. F., Hernquist, L., Cox, T. J., et al. 2006, ApJS, 163, 1

Hopkins, P. F., Hernquist, L., Cox, T. J., \& Kereš, D. 2008, ApJS, 175, 356

Hopkins, P. F., \& Quataert, E. 2010, MNRAS, 407, 1529

Jordi, K., Grebel, E. K., \& Ammon, K. 2006, A\&A, 460, 339
Karouzos, M., Jarvis, M. J., \& Bonfield, D. 2014, MNRAS, 439, 861

Kocevski, D. D., Faber, S. M., Mozena, M., et al. 2012, ApJ, 744, 148

Kormendy, J., \& Ho, L. C. 2013, ARA\&A, 51, 511

Krist, J. E., Hook, R. N., \& Stoehr, F. 2011, Proc. SPIE, 8127, 81270J

Lambas, D. G., Tissera, P. B., Alonso, M. S., \& Coldwell, G. 2003, MNRAS, 346, 1189

Lotz, J. M., Jonsson, P., Cox, T. J., \& Primack, J. R. 2010, MNRAS, 404, 575

LSST Dark Energy Science Collaboration 2012, arXiv:1211.0310

Man, A. W. S., Toft, S., Zirm, A. W., Wuyts, S., \& van der Wel, A. 2012, ApJ, 744,85

Matsuoka, Y., Strauss, M. A., Price, T. N., III, \& DiDonato, M. S. 2014, ApJ, 780,162

Momcheva, I. G., Brammer, G. B., van Dokkum, P. G., et al. 2016, ApJS, 225,27

Newton, R. D. A., \& Kay, S. T. 2013, MNRAS, 434, 3606

Pâris, I., Petitjean, P., Aubourg, E., et al. 2018, A\&A, 613, A51

Pâris, I., Petitjean, P., Ross, N. P., et al. 2017, A\&A, 597, A79

Peng, C. Y., Ho, L. C., Impey, C. D., \& Rix, H.-W. 2002, AJ, 124, 266

Richards, G. T., Strauss, M. A., Fan, X., et al. 2006, AJ, 131, 2766

Satyapal, S., Ellison, S. L., McAlpine, W., et al. 2014, MNRAS, 441, 1297

Schawinski, K., Treister, E., Urry, C. M., et al. 2011, ApJL, 727, L31

Schneider, D. P., Richards, G. T., Hall, P. B., et al. 2010, AJ, 139, 2360

Sérsic, J. L. 1963, BAAA, 6, 41

Shlosman, I., Frank, J., \& Begelman, M. C. 1989, Natur, 338, 45

Silverman, J. D., Kampczyk, P., Jahnke, K., et al. 2011, ApJ, 743, 2

Sirianni, M., Jee, M. J., Benítez, N., et al. 2005, PASP, 117, 1049

Skelton, R. E., Whitaker, K. E., Momcheva, I. G., et al. 2014, ApJS, 214, 24

Spacek, A., Scannapieco, E., Cohen, S., Joshi, B., \& Mauskopf, P. 2016, ApJ, 819,128

Springel, V., Di Matteo, T., \& Hernquist, L. 2005, MNRAS, 361, 776

Treister, E., Schawinski, K., Urry, C. M., \& Simmons, B. D. 2012, ApJL, 758, L39

Véron-Cetty, M.-P., \& Véron, P. 2010, A\&A, 518, A10

Villforth, C., Hamilton, T., Pawlik, M. M., et al. 2017, MNRAS, 466, 812

Weston, M. E., McIntosh, D. H., Brodwin, M., et al. 2017, MNRAS, 464, 3882

White, R. L., Becker, R. H., Helfand, D. J., \& Gregg, M. D. 1997, ApJ, 475, 479

Wright, E. L., Eisenhardt, P. R. M., Mainzer, A. K., et al. 2010, AJ, 140, 1868

Yue, M., Jiang, L., Shen, Y., et al. 2018, ApJ, 863, 21 